Meta

Journal des traducteurs

Translators' Journal

\title{
L'enseignement de la traduction au Canada
}

\section{Geneviève Mareschal}

Volume 50, numéro 1, mars 2005

Enseignement de la traduction dans le monde

Teaching Translation Throughout the World

URI : https://id.erudit.org/iderudit/010672ar

DOI : https://doi.org/10.7202/010672ar

Aller au sommaire du numéro

Éditeur(s)

Les Presses de l'Université de Montréal

ISSN

0026-0452 (imprimé)

1492-1421 (numérique)

Découvrir la revue

Citer cet article

Mareschal, G. (2005). L'enseignement de la traduction au Canada. Meta, 50(1), 250-262. https://doi.org/10.7202/010672ar

\section{Résumé de l'article}

Issue de l'adoption de lois linguistiques à la fin des années 60, la formation de traducteurs professionnels au Canada a pendant longtemps été axée sur les seules combinaisons anglais-français, français-anglais, principalement au premier cycle universitaire. Cette formation s'est par la suite étendue aux trois cycles d'études et s'est diversifiée tant dans sa forme que dans ses contenus. L'introduction progressive de spécialisations, de nouvelles combinaisons de langues, de programmes courts d'initiation, de programmes longs de recherche, de formations combinées, d'options coop, d'enseignements par Internet, a donné à ce secteur de l'enseignement universitaire un dynamisme et un allant garants de son avenir.
Ce document est protégé par la loi sur le droit d'auteur. L'utilisation des services d'Érudit (y compris la reproduction) est assujettie à sa politique d'utilisation que vous pouvez consulter en ligne.

https://apropos.erudit.org/fr/usagers/politique-dutilisation/ 


\title{
L'enseignement de la traduction au Canada
}

\author{
GENEVIÈVE MARESCHAL \\ Université d'Ottawa, Ottawa, Canada \\ gmaresch@uottawa.ca
}

\begin{abstract}
RÉSUMÉ
Issue de l'adoption de lois linguistiques à la fin des années 6o, la formation de traducteurs professionnels au Canada a pendant longtemps été axée sur les seules combinaisons anglais-français, français-anglais, principalement au premier cycle universitaire. Cette formation s'est par la suite étendue aux trois cycles d'études et s'est diversifiée tant dans sa forme que dans ses contenus. L'introduction progressive de spécialisations, de nouvelles combinaisons de langues, de programmes courts d'initiation, de programmes longs de recherche, de formations combinées, d'options coop, d'enseignements par Internet, a donné à ce secteur de l'enseignement universitaire un dynamisme et un allant garants de son avenir.
\end{abstract}

\begin{abstract}
A by-product of linguistic legislation voted in the late sixties, the training of professional translators in Canada has long been centered solely on the English-French FrenchEnglish linguistic combination, mainly at the undergraduate level. Over the years this training has spread over the three university levels and has changed in form as well as content. The progressive introduction of specializations, new language combinations, short initiation programs, long research programs, combined degrees, co-op options, distance education via the Internet, has created a very dynamic and fast-growing university sector.
\end{abstract}

\section{MOTS-CLÉS/KEYWORDS}

traduction professionnelle, programmes de traduction, formation de traducteurs, stages en traduction, traductologie

La création de programmes universitaires de traduction ${ }^{1}$ au Canada remonte à la fin des années 60. C'est l'Université de Montréal, bientôt suivie par l'Université Laval à Québec et par l'Université d'Ottawa, qui a mis sur pied le premier programme universitaire complet d'enseignement de la traduction professionnelle, sous la forme d'un baccalauréat spécialisé ${ }^{2}$ de premier cycle d'une durée de trois ans.

Très rapidement, d'autres universités ont suivi leurs traces et, à la fin des années 70, neuf baccalauréats de traduction avaient vu le jour. Réparti géographiquement entre la province du Manitoba à l'ouest et celle du Nouveau-Brunswick à l'est, le gros contingent des programmes se situait - et se situe encore - au Québec et en Ontario.

Cette brusque multiplication des programmes de traduction répondait à une demande issue d'un vaste mouvement législatif en matière linguistique, tant au fédéral qu'au provincial, qui allait avoir des retombées importantes pour la traduction. Adoptée en 1969 par le Parlement canadien, la Loi sur les langues officielles institue un bilinguisme officiel au fédéral en accordant des droits égaux à l'anglais et au français. La fonction publique fédérale se voit désormais obligée de fournir des services bilingues à l'ensemble de la population canadienne. Quelques années plus tard, en 1982, 
la Charte canadienne des droits et libertés viendra inscrire ce principe dans la Constitution. Au provincial, la Charte de la langue française consacre en 1977 le français comme la langue officielle du Québec. Le français devient de ce fait la langue normale et habituelle du travail, de l'enseignement, des communications, des affaires, de la législation, de la justice, et de tout l'appareil public québécois. L'anglais ayant prédominé jusqu'alors dans certains secteurs d'activité de la société québécoise, l'impact de cette loi sur les besoins de traduction n'allait pas tarder à se faire sentir. Ailleurs au Canada, l'adoption de lois linguistiques ou la reconnaissance de droits linguistiques, entre autres au Nouveau-Brunswick, en Ontario et au Manitoba, allait faire progresser le bilinguisme législatif, judiciaire et administratif de façon significative et favoriser l'essor de la traduction et des programmes de traduction.

Le développement de la traduction au Canada, à la fois comme profession, comme industrie et comme programme d'études, est donc issu de facteurs principalement socio-politico-linguistiques et non pas, comme on pourrait s'y attendre, de facteurs purement économiques et commerciaux. Cette origine politique se trouve corroborée par le fait qu'au Canada, $80 \%$ des revenus de l'industrie de la traduction sont liés, directement ou indirectement, au bilinguisme institutionnel et non pas au simple jeu de la concurrence commerciale, et que près de $50 \%$ de la demande de services de traduction provient du secteur public ${ }^{3}$. Il ne fait aucun doute que l'industrie de la traduction au Canada serait très différente - et probablement moins importante - si elle n'était pas conditionnée par l'environnement législatif canadien en matière de langues.

Cette origine se reflète aussi dans la façon dont les programmes de traduction se sont implantés au Canada et se sont construit une identité propre.

\section{Lieux d'implantation des programmes de traduction ${ }^{4}$}

Les programmes de traduction se sont développés et implantés dans les provinces canadiennes où le bilinguisme anglais-français était soit officiellement reconnu, soit fortement présent. Les deux provinces ayant opté pour un bilinguisme officiel sont le Nouveau-Brunswick et le Manitoba: dans ces deux provinces, un baccalauréat spécialisé en traduction a été instauré dès les années 70. Au Québec et en Ontario, c'est la coexistence de l'anglais et du français sur le territoire en raison de facteurs historiques et commerciaux et les retombées de la mise en œuvre des législations linguistiques sur le plan des ressources humaines qui ont favorisé la création des programmes. Montréal, important pôle économique de l'est du Canada, a toujours été fortement bilingue, et ce bilinguisme est une condition garante de son succès économique. Par ailleurs, l'important travail de francisation qui allait résulter de l'adoption de la Charte de la langue française au Québec a mobilisé pendant des années, et mobilise encore, un nombre élevé de traducteurs dans tous les secteurs d'activité. En Ontario, c'est à Ottawa que «les choses se passent». Capitale fédérale et siège du gouvernement du Canada, il s'y est développé une intense activité traductionnelle depuis l'adoption de la loi sur les langues officielles. Le Bureau de la traduction, organisme officiellement responsable de la traduction au sein du gouvernement canadien et pendant longtemps fournisseur exclusif des services de traduction aux ministères et organismes fédéraux, emploie à lui seul plus de mille traducteurs, terminologues et interprètes, et est de ce fait le plus gros employeur de traducteurs au Canada. Il n'est 
donc pas surprenant que ce soit dans ces deux provinces, le Québec et l'Ontario, que l'on trouve le plus grand nombre d'écoles et de programmes de traduction.

\section{Conception des programmes autour de deux langues}

Le marché de la traduction au Canada étant axé sur l'anglais et le français, les programmes de traduction ont été conçus autour de ces deux langues, et souvent de ces deux langues seulement. Cette conception de la formation à la traduction professionnelle va à l'encontre de la tradition européenne, par exemple, où les programmes exigent des candidats la connaissance d'au moins trois langues, sinon quatre, et offrent une formation dans de nombreuses combinaisons linguistiques. Pendant longtemps et encore maintenant dans certaines universités, les seules combinaisons offertes par les programmes sont anglais-français, français-anglais. L'introduction d'une troisième langue dans les programmes, généralement l'espagnol, est un phénomène récent attribuable à la ratification de traités de libre-échange avec certains pays d'Amérique latine et à l'accroissement des échanges qui en a résulté.

\section{Prédominance de la filière francophone}

La démographie canadienne, qui est constituée grosso modo de $70 \%$ d'anglophones et de $30 \%$ de francophones, a pour effet que les communications se font d'abord et principalement en anglais et que le gros de la demande de traduction se situe vers le français. Il s'ensuit que la majorité des programmes sont orientés vers le français et visent les candidats ayant le français comme langue dominante. Il a fallu attendre plusieurs années avant que des programmes pour anglophones, c'est-à-dire orientés vers l'anglais, soient établis. Ces programmes sont d'ailleurs peu nombreux: seules 3 universités sur 14 ont un baccalauréat distinct axé sur la combinaison françaisanglais. Les étudiants des programmes de traduction au Canada sont donc majoritairement des francophones inscrits dans la combinaison anglais-français.

La prédominance du français sur le marché de la traduction a aussi influencé la conception des cursus, où la traduction de l'anglais vers le français (composante version) constitue l'élément central des apprentissages. Le passage actif vers l'anglais (composante thème) n'occupe pas une place importante dans la formation. Suivant les programmes, il peut faire l'objet de quelques cours, parfois facultatifs, ou en être totalement absent.

\section{Similitude des programmes}

Conçus pour répondre à des besoins de bilinguisation officielle et visant essentiellement le marché national, les programmes de formation de base en traduction au Canada sont tous plus ou moins coulés dans le même moule. Les baccalauréats spécialisés présentent les mêmes composantes ${ }^{5}$ dans des proportions qui peuvent évidemment varier, et ont tous pour objectif de former de bons traducteurs généralistes. Les diplômés des universités ont donc une formation très comparable et, si un diplômé se démarque d'un autre, c'est plus en raison d'aptitudes et de connaissances personnelles que de grosses disparités dans la formation.

Trente ans plus tard, qu'en est-il de l'enseignement de la traduction? 


\section{Quatorze institutions dispensent une formation en traduction}

Treize universités et un établissement post-secondaire dispensent actuellement des formations structurées en traduction, terminologie ou interprétation. Ces institutions sont, dans l'ordre alphabétique, l'Université Carleton, l'Université Concordia (à Montréal), l'Université Laurentienne (à Sudbury), l'Université Laval (à Québec), l'Université McGill (à Montréal), l'Université de Moncton, l'Université de Montréal, l'Université d'Ottawa, l'Université du Québec en Outaouais (à Gatineau), l'Université du Québec à Trois-Rivières, le Collège universitaire de Saint-Boniface, l'Université de Sherbrooke, et le Collège Glendon de l'Université York (à Toronto) ${ }^{6}$. Onze universités (Concordia, la Laurentienne, Laval, McGill, Moncton, Montréal, Ottawa, Québec en Outaouais, Québec à Trois-Rivières, Saint-Boniface, York) offrent des formations complètes menant à un grade universitaire et ouvrant droit au titre professionnel de traducteur, terminologue ou interprète agréé décerné par les associations professionnelles. Deux universités (Carleton et Sherbrooke) se limitent à des formations courtes d'initiation à la traduction. Quant au seul établissement post-secondaire, le Vancouver Community College, il forme des interprètes dits «communautaires", appelés à travailler dans les milieux hospitaliers, les cours de justice et les tribunaux administratifs. À l'exception de ce dernier établissement, toutes les universités offrant des programmes de traduction sont regroupées au sein de l'Association canadienne des écoles de traduction (ACET), fondée en 1973 et qui leur sert de lieu d'échanges.

Au niveau universitaire, les unités administratives responsables des programmes de traduction sont généralement intégrées à une faculté ou à un collège. Elles peuvent y être entièrement autonomes et avoir le plein statut de département, et portent alors souvent le nom d' «école» à cause de l'orientation professionnelle de leurs programmes; c'est le cas, par exemple, de l'École de traduction et d'interprétation de l'Université d'Ottawa. Le plus souvent, les programmes de traduction sont associés à une discipline connexe au sein d'un département. Ainsi, la traduction à l'Université de Montréal partage sa structure administrative avec la linguistique au sein du Département de linguistique et traduction. Parfois, les programmes de traduction sont rattachés à un département déjà existant, comme c'est le cas à l'Université Concordia où la traduction fait partie du Département d'études françaises. Cette diversité de désignations reflète des préoccupations essentiellement administratives et structurelles, l'autorité pédagogique des programmes résidant normalement dans la discipline.

Par rapport aux années 70, le nombre d'établissements dispensant une formation en traduction est resté relativement stable; en effet, seules trois nouvelles universités sont venues grossir les rangs de l'ACET: ce sont les universités du Québec en Outaouais, de Sherbrooke et Carleton. Ces trois universités sont situées au Québec et en Ontario, comme la majorité des autres institutions. Un seul nouveau programme a vu le jour dans une autre province, le programme d'interprétation communautaire du Vancouver Community College, qui est établi en Colombie-Britannique. C’est la forte immigration en provenance du continent asiatique qui est à l'origine de l'établissement de ce programme dans cette province. 


\section{L'enseignement de la traduction s'est étendu aux trois cycles universitaires}

Une des principales caractéristiques de l'évolution de la formation en traduction au Canada réside dans la création de programmes à tous les degrés universitaires et dans l'introduction de formations courtes ou accélérées.

Au premier cycle, le baccalauréat spécialisé est et demeure le programme professionnel par excellence, axé directement sur le marché de la traduction. Conformément aux normes des programmes d'études au Canada, sa durée varie de trois à quatre ans et le nombre de crédits universitaires requis pour obtenir le diplôme est de 90 à 120 crédits $^{7}$. Des formations accélérées, sur deux ans, sont également offertes dans certains établissements et s'adressent aux étudiants inscrits à une autre spécialisation ou ayant déjà un premier diplôme. Le but de ces programmes est de former des traducteurs généralistes compétents, rapidement opérationnels sur le marché du travail. Diverses formations courtes, ne conduisant pas à un grade universitaire, sont également disponibles au premier cycle.

Au deuxième cycle, divers programmes se sont développés au cours des années dans un certain nombre d'universités (Montréal, Ottawa, Laval, York, Concordia, Université du Québec en Outaouais). Leur contenu, leur durée, leur orientation et leur appellation varient grandement.

On trouve à ce niveau des programmes de traduction de type professionnel, destinés à des étudiants ayant déjà un premier diplôme universitaire et souhaitant acquérir une formation en traduction, qui leur permettra d'exploiter différemment les compétences qu'ils ont acquises dans une autre discipline. On y trouve aussi des programmes permettant d'ajouter une spécialité à une formation préalablement acquise en traduction. Ainsi, la spécialisation en localisation, en terminologie, dans un domaine particulier du savoir (par exemple en traduction juridique ${ }^{8}$ ), ou encore dans une troisième langue, fait généralement partie des programmes de deuxième cycle. C'est au deuxième cycle aussi que se situe la formation à l'interprétation de conférence. Cette formation, qui s'appuie sur une première formation universitaire, souvent en traduction mais aussi dans d'autres disciplines, et à laquelle vient idéalement s'ajouter une expérience de travail en traduction, est fort peu représentée dans les programmes au Canada. Bien que ceux-ci soient annoncés par au moins deux universités, un seul programme n'est véritablement actif dans le domaine, celui de l'Université d'Ottawa, qui forme des interprètes uniquement dans la combinaison anglais-français/français-anglais. La durée de ces programmes est normalement d'une année universitaire.

À côté de ces programmes professionnels existent des programmes de maîtrise orientés vers la recherche. Les cours sont de nature plus théorique et visent, d'une part, à développer chez l'étudiant une connaissance générale des grands champs de la discipline traductologique et, d'autre part, à le spécialiser dans le domaine de recherche qui fera l'objet de sa thèse. Il faut généralement compter deux ans pour terminer une maîtrise de recherche.

Au troisième cycle, deux universités offrent des programmes de doctorat en traduction ou en traductologie, l'Université de Montréal et l'Université d'Ottawa. Ces programmes sont, de par leur nature, axés sur la recherche et donnent lieu à des travaux d'envergure dans le vaste champ de la traductologie. 


\section{Les programmes se sont diversifiés}

On constate depuis quelques années une certaine diversification dans les formations en traduction et dans les façons de dispenser ces formations.

Des programmes courts d'initiation à la traduction professionnelle ou à la rédaction professionnelle, généralement de 30 crédits, ont été instaurés au premier cycle ou dans le cadre de la formation permanente. Créés sous forme de certificats autonomes, de mineures ou de concentrations à l'intérieur d'un baccalauréat spécialisé dans une discipline autre que la traduction, ces programmes courts attirent un nombre important de candidats qui y voient l'occasion de sanctionner par un diplôme une pratique occasionnelle de la traduction et de parfaire celle-ci, ou encore d'ajouter une nouvelle corde à leur arc. Ces programmes constituent aussi un bon tremplin vers la poursuite d'études plus complètes au baccalauréat.

La formation à la traduction dans des combinaisons autres qu'anglais-français est aussi dispensée sous la forme de programmes courts. C'est le cas des certificats de traduction espagnol-français et allemand-français offerts par l'Université de Montréal. Les diplômes d'études supérieures de deuxième cycle de type professionnel illustrent également cette diversification.

La diversification s'applique aussi à la combinaison de savoirs proches mais distincts à l'intérieur des programmes. C'est le cas, par exemple, de la combinaison traduction-rédaction dans le programme de baccalauréat en traduction et en rédaction de l'Université du Québec en Outaouais. La rédaction professionnelle et technique est d'ailleurs de plus en plus souvent «chapeautée» par les écoles de traduction, comme à l'Université York, où le certificat de rédaction technique et professionnelle relève de l'École de traduction.

Diversification aussi dans les «façons d'enseigner»: le certificat de traduction anglais-français/français-anglais sur Internet du Collège universitaire de Saint-Boniface, qui permet de suivre un cours d'initiation à la traduction de 30 crédits à partir de chez soi, est un bon exemple d'adaptation aux besoins de la clientèle, tant sur le plan méthodologique que sur celui du cadre d'apprentissage 9 .

\section{Contenu des programmes}

L'objectif des programmes étant de former des traducteurs généralistes compétents et rapidement opérationnels sur le marché, c'est en termes de compétences et de savoir-faire à acquérir et à développer que la formation peut se définir.

\section{Compétences linguistiques}

Ces compétences concernent la connaissance pratique et approfondie des langues à partir desquelles et vers lesquelles le traducteur va traduire. Bien que les langues de la combinaison linguistique choisie doivent être maîtrisées avant l'admission au programme, les études de traduction comportent toutes des cours de perfectionnement et de renforcement linguistique. Il s'agit principalement de cours avancés de français, d'anglais et, s'il y a lieu, d'espagnol, axés sur la langue écrite et conçus dans l'optique de la traduction. 


\section{Compétences traductionnelles}

Il s'agit ici de donner à l'étudiant les compétences nécessaires, c'est-à-dire les techniques, méthodes, moyens et outils, pour effectuer le passage d'une langue à l'autre dans le respect des normes établies et imposées par la profession. L'acquisition de ces compétences constitue l'élément fondamental de la formation et la raison d'être d'un programme universitaire distinct en traduction. Des exercices nombreux et variés, portant sur divers types de textes et accompagnés d'une analyse des difficultés rencontrées et d'une réflexion sur le processus traductionnel visent à atteindre ce but. Les cours de traduction générale, de traduction spécialisée, de méthodologie de la traduction contribuent directement à l'acquisition de ces compétences.

\section{Compétences rédactionnelles}

L'importance de l'écrit en traduction oblige à fournir à l'étudiant les outils nécessaires pour rédiger un texte général ou spécialisé de qualité. Ces compétences rédactionnelles sont le complément indispensable des compétences linguistiques et des compétences traductionnelles. Elles doivent permettre au traducteur d'exprimer avec clarté, précision et justesse un contenu donné dans une langue donnée, en respectant les caractéristiques inhérentes au texte et à la langue. Les cours de rédaction et de révision favorisent ces acquisitions.

\section{Compétences thématiques}

Idéalement, les programmes de traduction devraient donner à l'étudiant des connaissances de base dans les grands champs de l'activité humaine (droit, économie, sociologie, etc.). La durée des études obligeant à concentrer les apprentissages sur l'acquisition des techniques de traduction (savoir-faire traductionnel), les enseignements thématiques sont le plus souvent intégrés aux cours de traduction spécialisée et peuvent être centrés sur un même domaine ou aborder divers sujets. Certains programmes ont opté pour la première approche et proposent des mini-spécialisations pouvant être acquises par le biais d'un groupe de cours axés sur un même domaine de connaissance (par exemple le droit, l'économie, les sciences médicales) et combinant l'étude des concepts, l'apprentissage de la langue de spécialité et la pratique de la traduction dans le domaine. L'autre option, axée sur divers sujets, vise à donner à l'étudiant un aperçu de l'immense variété des domaines et des textes à traduire et à lui faire développer des méthodes de travail qui lui permettront de faire face intelligemment à la polyvalence thématique requise de sa part.

\section{Compétences de recherche}

L'étudiant doit acquérir les méthodes et techniques de recherche nécessaires pour trouver l'information et la terminologie dont il a besoin pour traduire un texte donné dans un domaine donné. Ces compétences sont essentielles au travail du traducteur. Elles garantissent la qualité de la traduction et visent à assurer l'autonomie et la polyvalence du traducteur. Des cours précis, entre autres de recherche documentaire et de terminologie, apportent à l'étudiant les bases méthodologiques et le jugement critique requis pour aller chercher l'information dont il a besoin. 


\section{Compétences informatiques}

Les outils informatiques d'aide à la traduction font de plus en plus partie de l'environnement professionnel du traducteur. Une initiation à ces outils est donc devenue incontournable dans la formation. Tous les programmes comportent au moins un cours, sinon plusieurs, sur les logiciels informatiques destinés aux traducteurs. Au départ, ces logiciels faisaient principalement l'objet de démonstrations et les connaissances exigées des étudiants portaient sur leurs principes de fonctionnement. Aujourd'hui, les écoles de traduction sont pour la plupart dotées de laboratoires informatiques multipostes et mettent à la disposition des étudiants des postes de travail équipés des principaux outils du traducteur, reproduisant les conditions de travail de celui-ci. Ces laboratoires qui peuvent servir de salles de classe et de salles de travail permettent aux étudiants de se familiariser hands-on avec les logiciels traductiques et terminotiques pertinents, tels que les logiciels de gestion de bases de données terminologiques, les mémoires de traduction, les banques de termes, les concordanciers, etc. Par ailleurs, les outils informatiques sont de plus en plus souvent intégrés directement aux cours: l'utilisation de logiciels de création de pages web et de présentation de type Powerpoint, d'aligneurs de texte et de bi-textes, de logiciels de dépouillement de termes, etc. est de plus en plus courante dans les cours de traduction, de terminologie et de rédaction.

À ces compétences viennent s'ajouter, dans tous les programmes, des cours plus théoriques, tels qu'histoire de la traduction, théorie de la traduction, stylistique comparée, qui favorisent une réflexion sur la traduction, sur ses méthodes, sur son évolution dans le temps et sur les traducteurs qui s'y sont illustrés.

\section{Les stages dans la formation}

Au Canada, des liens étroits se sont tissés au cours des ans entre les universités et le milieu professionnel de la traduction. La concrétisation de ces liens se manifeste par la participation active des professionnels à la formation pratique des étudiants par le biais des stages. Connu sous diverses appellations - stage pratique, stage en entreprise, stage en milieu de travail, stage de perfectionnement, etc. -, le stage est la composante résolument pratique de la formation. Activité fortement encouragée par les responsables des programmes, le stage désigne l'expérience acquise sur le marché du travail en traduisant des vrais textes destinés à des vrais clients/utilisateurs sous la supervision d'un professionnel compétent. Il permet à l'étudiant d'avoir un contact réel avec le monde professionnel et de mettre à l'épreuve les compétences et connaissances qu'il a acquises dans le cadre de ses études. Intégré à tous les programmes complets de traduction, le stage, qui est tributaire des disponibilités offertes sur le marché du travail, peut être soit obligatoire, soit facultatif.

Pour permettre au plus grand nombre d'étudiants d'avoir accès à cette expérience professionnelle, différentes formules de stage ont été développées par les universités. Un tour d'horizon de ces formules permettra d'apprécier le caractère pragmatique et souvent innovateur des stages. Notons que, parmi les critères essentiels à la reconnaissance d'un stage, figurent au premier rang le type de travail confié au stagiaire et l'encadrement didactique de celui-ci.

La formule classique est le stage externe, où l'étudiant passe un certain nombre de jours de stage dans un cabinet de traduction ou dans le service de traduction d'une 
(grande) entreprise. Ce stage se déroule souvent l'été, soit en dehors de la session universitaire proprement dite, et sa durée, qui comporte une période minimale, peut varier en fonction de divers facteurs dont les principaux sont le volume de travail disponible et la possibilité d'encadrement par l'employeur. L'intérêt de ce stage est de permettre au candidat-traducteur de participer activement à la vie d'une entreprise de traduction, de se familiariser avec sa structure et son fonctionnement, et d'apprendre à connaître les exigences et les réalités du marché.

L'industrie canadienne de la traduction se caractérisant par un très grand nombre de traducteurs indépendants et de (très) petites entreprises (plus de $80 \%$ ), l'offre de stages externes est nettement inférieure à la demande. Pour pallier cette pénurie, deux autres formules de stage ont été développées au cours des années: le stage interne et le stage en partenariat.

Le stage interne, aussi appelé cabinet étudiant, reproduit au sein de l'université un cabinet de traduction, composé en l'occurrence d'apprentis traducteurs et entièrement géré par eux, sous la supervision d'un professeur/traducteur professionnel. Toutes les activités, fonctions, responsabilités d'un cabinet de traduction sont mises en œuvre et prises en charge par les étudiants, qu'il s'agisse de la recherche de clients, de la facturation des travaux, de la répartition du travail et des heures de permanence, de la mise à jour des fichiers terminologiques, de l'archivage des textes dans la mémoire de traduction, de l'exécution des travaux, du respect des délais, sans oublier le suivi des plaintes. Comptabilisable en nombre de semaines, en nombre de mots à traduire, relire ou réviser, ou encore en une combinaison semaines/mots/heures de présence, ce stage permet de sensibiliser les étudiants à la réalité et aux conditions de travail des traducteurs indépendants ou pigistes. Il développe leur sens de l'initiative, les oblige à gérer leur projet de traduction et leur temps, et les responsabilise face à la livraison d'un produit fini et de qualité.

Le stage en partenariat, modèle de stage le plus récent, créé à l'instigation du Bureau de la traduction du gouvernement fédéral, propose le jumelage d'un étudiant avec un professionnel de la traduction membre d'un service ou cabinet de traduction, ou avec un traducteur indépendant, principalement via l'Internet. Ce stage, qui se déroule pendant la session d'études parallèlement aux autres cours du programme, impose à l'étudiant de traduire de 700 à 1000 mots par semaine pendant toute la durée de la session. Les textes lui sont fournis par le traducteur avec lequel il est jumelé, qui assure également la révision et l'encadrement de l'étudiant. L'Internet sert de support principal au processus d'échange de textes, de révision, de corrections à apporter, de relecture, ainsi qu'aux commentaires pédagogiques, et permet à l'étudiant de se familiariser avec une autre composante de la réalité professionnelle, celle du «tout en ligne».

Les trois formules de stage qui viennent d'être décrites sont de type court. Une autre formule, de type long, le stage dit «coop», a été empruntée à d'autres programmes universitaires à vocation professionnelle (comptabilité, sciences infirmières, etc.) et développée avec succès pour la traduction par diverses universités ${ }^{10}$. Le stage coop s'insère en fait dans le cadre d'un programme coopératif, ou d'alternance travailétudes, dans lequel l'étudiant suit successivement une session d'études de quatre mois et une session de stage de quatre mois, et ce pendant toute la durée de son programme d'études. La durée de sa formation s'en trouve rallongée, mais au terme de son programme, l'étudiant peut justifier d'une année complète d'expérience pro- 
fessionnelle. Les stages coop sont effectués en entreprise et sont obligatoirement rémunérés.

L'apport des stages dans un programme de traduction est substantiel et profite à tous les partenaires. L'étudiant y fait l'apprentissage de la réalité professionnelle, y compris des aspects de cette réalité auxquels les programmes universitaires peuvent difficilement former, tels que la vitesse, le rendement et l'autonomie, et il a l'occasion de faire valoir ses compétences. L'employeur, lui, se trouve dans la situation privilégiée d'avoir, à peu de frais, un employé de plus et, s'il est en période de recrutement, de pouvoir tester à l'avance un certain nombre de candidats avant de faire son choix. Quant à l'université, les stages en entreprise constituent un excellent moyen de renforcer ses liens avec le milieu professionnel, de se tenir au courant de l'évolution de la profession et du marché, et partant, de mettre à jour ses programmes et d'améliorer le produit qu'elle diplôme.

\section{La recherche en traduction}

Avec le développement des études de deuxième et de troisième cycle, la recherche en traduction a véritablement pris son essor. Les liens que la traduction entretient avec les autres disciplines linguistiques et langagières, sa présence dans tous les secteurs de l'activité humaine et son rôle dans l'histoire de l'humanité et dans la transmission des idées éclairent l'étendue du champ de recherche de la discipline traductologique. Il suffit de jeter un coup d'œil aux titres des thèses de maîtrise et de doctorat pour voir combien les travaux et les intérêts de recherche sont variés. De la traduction scientifique et technique à la traduction littéraire, en passant par la terminologie, la traduction automatique, l'interprétation, l'histoire de la traduction, la pédagogie de la traduction, la lexicographie bilingue, la réception des traductions à l'étranger, etc., les sujets et domaines à explorer sont infinis.

Les universités disposent aussi d'une bonne assise de diffusion des résultats de la recherche traductologique, avec deux revues savantes bien établies et connues internationalement: Meta, revue du Département de linguistique et de traduction de l'Université de Montréal, et TTR (Traduction, Terminologie, Rédaction), revue officielle de l'Association canadienne de traductologie. Par ailleurs, les Presses de l'Université d'Ottawa comptent deux collections consacrées à la traduction et à la traductologie: Regards sur la traduction et Pédagogie de la traduction. En dehors du milieu universitaire, le Bureau de la traduction du gouvernement fédéral, avec sa revue L'Actualité terminologique, et l'Office québécois de la langue française avec Terminogramme sont également de bons disséminateurs des travaux de recherche en terminologie. Les associations professionnelles ne sont pas en reste: la revue Circuit de l'Ordre des traducteurs, terminologues et interprètes agréés du Québec (OTTIAQ) offre aux traducteurs quatre fois par an des dossiers et des renseignements du plus haut intérêt sur la profession.

\section{Tendances actuelles de la profession et leur impact sur la formation}

Dans le rapport final de l'enquête menée sur la situation de la traduction au Canada à la fin des années 90, le Comité sectoriel de l'industrie canadienne de la traduction a fait ressortir un certain nombre de tendances auxquelles les institutions de formation sont actuellement confrontées. 


\section{Technologisation de la profession et des processus de traduction}

Les dernières années ont été marquées par l'introduction sur le marché de la traduction d'outils informatiques sans cesse plus nombreux, plus sophistiqués et plus performants. Le travail du traducteur et les processus traductionnels s'en trouvent chaque jour un peu plus modifiés.

Il ne fait aucun doute que la formation doive prendre en compte cette évolution de la profession et ces nouvelles exigences, et elle le fait. Les technologies reliées à la traduction sont pleinement intégrées dans les enseignements dispensés au futur traducteur. Le défi qui se pose toutefois aux universités est de se tenir à jour par rapport à cette évolution. Ce défi est d'abord d'ordre financier: le coût élevé des laboratoires et des équipements matériels et logiciels exige des investissements auxquels les écoles et départements de traduction ne sont pas habitués. Ce défi est ensuite d'ordre professoral: le recrutement de professeurs spécialisés dans les outils informatiques d'aide à la traduction - des oiseaux rares - s'avère difficile. Un certain nombre d'universités en a fait l'expérience récemment en tentant vainement de recruter de jeunes diplômés ou chercheurs dans le domaine.

Sur une note plus positive, les étudiants sont de mieux en mieux équipés sur le plan informatique, et les fonds actuellement investis dans les laboratoires pourront vraisemblablement être un jour reconvertis et servir à alimenter en logiciels traductiques et terminotiques les étudiants qui travailleront à partir de chez eux.

\section{La mondialisation et l'ouverture au multilinguisme}

L'internationalisation des échanges et l'ouverture des marchés mondiaux font aujourd'hui partie des réalités quotidiennes. L'avènement de l'Internet et son utilisation à l'échelle planétaire ont rendu cette réalité encore plus perceptible. Mais la conquête d'un marché passe obligatoirement par l'apprivoisement de la langue et de la culture. Bien qu'encore largement autosuffisante sur le marché national, l'industrie canadienne de la traduction doit étendre ses services à d'autres combinaisons de langues que le français et l'anglais si elle veut se positionner sur le marché mondial. La diversification des langues devient donc une des conditions garantes de succès pour l'avenir. Quand on sait le peu d'importance accordé traditionnellement au Canada à l'enseignement de langues autres que le français et l'anglais, le travail de persuasion à accomplir tant auprès des administrateurs universitaires qu'auprès des étudiants n'est pas une mince tâche, d'autant plus que les langues en demande sont le japonais, le coréen et le chinois.

Certes, on ne peut que se réjouir des efforts entrepris par certaines universités pour intégrer l'espagnol et l'allemand dans leurs programmes, mais il reste encore beaucoup de chemin à accomplir avant de pouvoir diversifier les combinaisons linguistiques et jouer un rôle sur le marché mondial. Une avenue intéressante à cet égard et de plus en plus exploitée est celle des échanges internationaux d'étudiants, qui favorisent l'acquisition d'autres langues et la familiarisation avec d'autres cultures.

\section{Formation de la relève}

Il y a pénurie de traducteurs sur tous les fronts, sur la scène nationale et internationale, et dans toutes les spécialités. Bien que garantissant des emplois rémunérateurs, 
les métiers de la traduction ne constituent pas un grand pôle d'attraction pour les jeunes générations. Un important travail de démarchage reste à faire, à plus forte raison si l'on veut attirer de bons potentiels. Par ailleurs, comme un leitmotiv, le discours des employeurs et des propriétaires de cabinets scande son besoin de traducteurs spécialisés et expérimentés. Conçus pour former des traducteurs généralistes, les programmes devront faire preuve d'initiative et d'innovation s'ils veulent répondre à la demande. La voie la plus rapide, que certaines universités ont déjà adoptée, est de recruter des diplômés d'autres disciplines et de leur donner une formation accélérée en traduction. Le défi reste toutefois d'aller chercher des diplômés dans les disciplines porteuses, telles que droit, économie, sciences médicales, biologie, etc.

Les universités ont pris acte des constats et des recommandations du Comité sectoriel de l'industrie canadienne de la traduction et ont déjà commencé à adapter leurs enseignements aux impératifs du marché, en instaurant de nouvelles combinaisons de langues dans les programmes, en développant des spécialisations telles que la localisation et la rédaction professionnelle, ou en intégrant pleinement les outils informatiques dans les apprentissages. Autant de signes encourageants qui témoignent d'une «industrie de la formation » vigoureuse, dynamique et ouverte au changement, qui ne néglige toutefois pas son profond attachement à la formation fondamentale.

\section{NOTES}

1. Il y avait bien quelques cours de traduction isolés dans les universités (la traduction professionnelle est enseignée depuis 1936 à Université d'Ottawa), mais pas de programme complet ni structuré.

2. Avant de s'appeler baccalauréat spécialisé, le diplôme décerné par l'Université de Montréal était une licence en traduction.

3. Ces données proviennent de l'enquête menée en 1998-1999 par le Comité sectoriel de l'industrie canadienne de la traduction sur la situation de la traduction, de la terminologie et de l'interprétation au Canada.

4. Sous traduction, nous englobons aussi la terminologie, l'interprétation et la rédaction professionnelle bilingue.

5. Pour une comparaison des composantes des programmes de traduction au Canada, voir l'article de Egan Valentine dans le collectif sur La formation à la traduction professionnelle, à paraître aux Presses de l'Université d'Ottawa en 2003.

6. Pour le détail des programmes par université, consulter le site de l'Association canadienne des écoles de traduction (ACET) à l'adresse suivante: <http://www.uottawa.ca/associations/acet $>$. Pour des renseignements sur les programmes d'interprétation communautaire du Vancouver Community College, voir à <http: //continuinged.vcc.ca/interpreting $>$.

7. La longueur des programmes spécialisés varie d'une province à l'autre, en fonction du régime universitaire. Au Québec, les études spécialisées de $1^{\text {er }}$ cycle universitaire comptent 90 crédits, les étudiants recevant une formation post-secondaire et pré-universitaire au sein des cégeps (collèges d'enseignement général et professionnel). Dans les autres provinces, les études spécialisées de $1^{\mathrm{er}}$ cycle comptent 120 crédits, la première année étant consacrée à la formation générale.

8. L'université d'Ottawa a dispensé pendant une dizaine d'années une formation spécialisée en traduction juridique au $2^{\mathrm{e}}$ cycle. Le programme a été suspendu en 1995 pour des raisons de coût et de ressources professorales, mais une remise sur pied de ce programme est actuellement à l'étude.

9. Pour une description détaillée de la formation sur Internet, voir les articles de Marie-Christine Aubin, entre autres dans le collectif sur La formation à la traduction professionnelle, Presses de l'Université d'Ottawa (2003).

10. C'est l'École de traduction et d'interprétation de l'Université d'Ottawa qui a mis sur pied le premier programme coop en 1987. Elle fut rapidement suivie par l'Université Concordia, puis plus récemment par l'Université de Montréal. 


\section{RÉFÉRENCES}

Aubin, M.-C. (2003): «Offrir des cours de traduction par l'Internet: les enjeux pédagogiques», dans Mareschal, G. et al. (dir.), La formation à la traduction professionnelle, Ottawa, Les Presses de l'Université d'Ottawa.

Circuit, 70, hiver 2001, numéro consacré en partie à la «Formation et technologie».

Comité sectoriel de l'industrie canadienne de la traduction (1999): L'industrie canadienne de la traduction. Stratégie de développement des ressources humaines et d'exportation, Ottawa.

Delisle, J. (1987): La traduction au Canada - Translation in Canada 1534-1984, Ottawa, Les Presses de l'Université d'Ottawa.

Valentine, E. (2003): «La modélisation des programmes existants comme moyen d'éclairage sur la formation en traduction", dans MAReschal, G. et al. (dir.), La formation à la traduction professionnelle, Ottawa, Les Presses de l'Université d'Ottawa. 